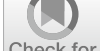

Check for

updates

Cite as

Nano-Micro Lett.

(2022) $14: 56$

Received: 28 October 2021

Accepted: 28 December 2021

Published online: 5 February 2022

(C) The Author(s) 2022

\section{Self-Assembly 3D Porous Crumpled MXene Spheres as Efficient Gas and Pressure Sensing Material for Transient All-MXene Sensors}

\author{
Zijie Yang ${ }^{1}$, Siyuan $\mathrm{Lv}^{1}$, Yueying Zhang ${ }^{1}$, Jing Wang ${ }^{2}$, Li Jiang ${ }^{1}$, Xiaoteng Jia ${ }^{1} \bowtie$, \\ Chenguang Wang ${ }^{1}$, Xu Yan ${ }^{1}$, Peng Sun ${ }^{1}$, Yu Duan ${ }^{1}$, Fangmeng Liu ${ }^{1}{ }^{凶}$, Geyu Lu${ }^{1}$
}

\title{
HIGHLIGHTS
}

- 3D porous crumpled MXene spheres were synthesized by ultrasonic spray pyrolysis technology.

- All-MXene transient sensors utilizing porous crumpled MXene sphere as sensing material and MXene films as electrodes were developed, which achieved excellent gas/pressure sensing performance.

- Both gas and pressure sensors can achieve rapid and controllable degradation in medical-grade $\mathrm{H}_{2} \mathrm{O}_{2}(2 \%)$ within $6 \mathrm{~h}$.

ABSTRACT Environmentally friendly degradable sensors with both hazardous gases and pressure efficient sensing capabilities are highly desired for various promising applications, including environmental pollution monitoring/prevention, wisdom medical, wearable smart devices, and artificial intelligence. However, the transient gas and pressure sensors based on only identical sensing material that concurrently meets the above detection needs have not been reported. Here, we present transient all-MXene $\mathrm{NO}_{2}$ and pressure sensors employing threedimensional porous crumpled MXene spheres prepared by ultrasonic spray pyrolysis technology as the sensing layer, accompanied with water-soluble polyvinyl alcohol substrates embedded with patterned MXene electrodes. The gas sensor achieves a ppb-level of highly selective $\mathrm{NO}_{2}$ sensing, with a response of up to $12.11 \%$ at $5 \mathrm{ppm}$

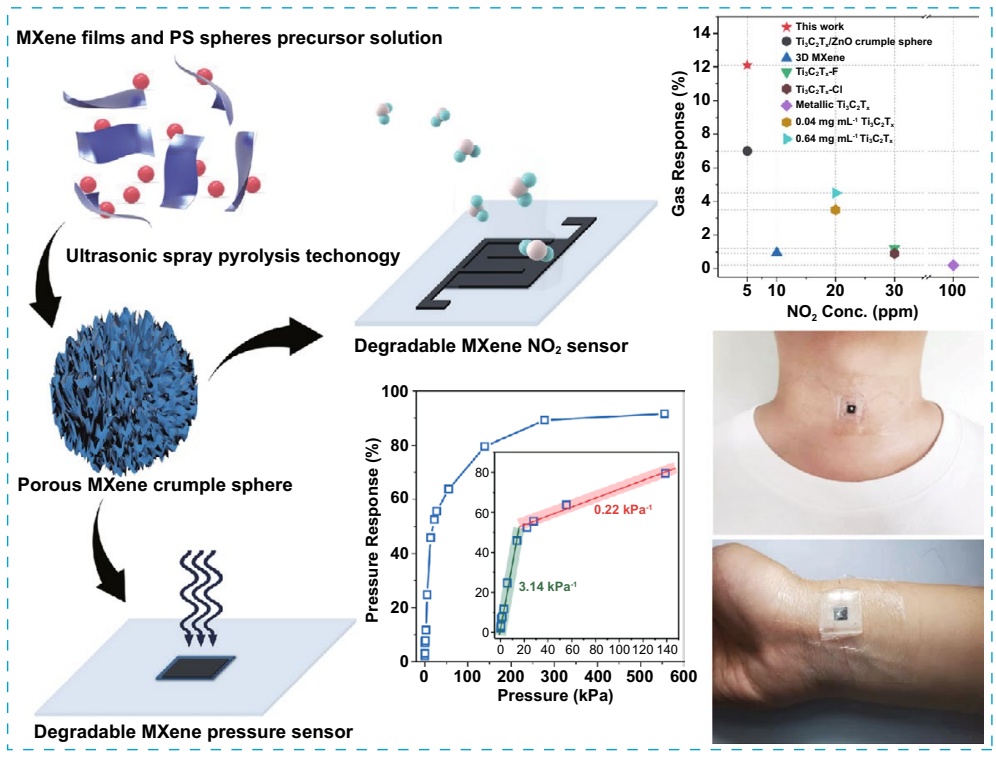
$\mathrm{NO}_{2}$ and a detection range of $50 \mathrm{ppb}-5 \mathrm{ppm}$, while the pressure sensor has an extremely wide linear pressure detection range of $0.14-22.22 \mathrm{kPa}$ and fast response time of $34 \mathrm{~ms}$. In parallel, all-MXene $\mathrm{NO}_{2}$ and pressure sensors can be rapidly degraded in medical $\mathrm{H}_{2} \mathrm{O}_{2}$ within $6 \mathrm{~h}$. This work provides a new avenue toward environmental monitoring, human physiological signal monitoring, and recyclable transient electronics.

KEYWORDS All-MXene; 3D porous crumpled MXene sphere; Transient; $\mathrm{NO}_{2}$ and pressure sensor

$\triangle$ Xiaoteng Jia, xtjia@jlu.edu.cn; Fangmeng Liu, liufangmeng@jlu.edu.cn

1 State Key Laboratory of Integrated Optoelectronics, College of Electronic Science and Engineering, Jilin University, 2699 Qianjin Street, Changchun 130012, People's Republic of China

2 School of Electronic and Information Engineering, Changchun University of Science and Technology, Changchun 130022,

People's Republic of China 


\section{Introduction}

In the Internet of Things era, wearable sensors as information receiving nodes have grown rapidly [1]. Wearable gas and pressure sensors, in particular, are useful for detecting hazardous gases and monitoring human physiological signals [2,3]. Meanwhile, transient and environmentally friendly devices that reduce the growing electronic waste are becoming crucial [4-6]. Therefore, the development of high-performance transient gas and pressure sensors has essential significance in wearable electronics.

Recently, transient gas and pressure sensors have been emerging [7, 8]. Jiang et al. developed a transient paperbased composite decorated with reduced graphene oxide and polyaniline with ammonia sensitivity and partial degradation [9]. Shen's group demonstrated a bacterial cellulose/MXene composite aerogel toward ammonia and pressure detection [10]. However, the above-mentioned sensors only achieved partial degradation when realizing gas/pressure sensing. In this regard, traditional composite-based sensor materials struggle to meet the demands of high gas and pressure sensitivity and complete device transiency. Gas and pressure sensors made of the same degradable sensing and electrode materials are ideal for achieving full transiency upon only one external trigger. MXenes $\left(\mathrm{Ti}_{3} \mathrm{C}_{2} \mathrm{~T}_{x}\right)$, as a novel class of two-dimensional nanomaterials with rich surface functional groups, have been identified as the sensing layer and electrode due to their high conductivity, excellent signal-tonoise ratio, and abundant hydroxyl on the surface, which is superior to other metal oxides and two-dimensional (2D) materials [11-18]. Meanwhile, because of their chemical instability, MXenes exhibit controllable transiency in $\mathrm{H}_{2} \mathrm{O}_{2}$ and $\mathrm{NaOH}$ aqueous solutions $[19,20]$. To achieve superior gas and pressure-sensing properties while maintaining complete degradation, it is necessary to tailor the architecture and composition of MXene sensing materials and ingeniously design the device architectures.

In MXene-based gas sensors, researchers have adopted different strategies to induce abundant oxygen terminals and high specific surface area, such as surface group modification [21, 22], microstructure design [23], and compounding with other nanomaterials [24], resulting in good sensitivity, selectivity, and low detection limits. In addition, the intercalation of various nanomaterials between MXene layers, such as silk fibroin [25, 26], cellulose nanofibers [19, 27], and holothurian-like microspheres [28], effectively enhances the piezoresistive properties. However, none of the above modification treatments are efficient toward the synergetic improvement of both the gas and pressure-sensing properties. Recent work has demonstrated that increasing the material porosity is a practical approach for boosting both gas and pressure sensing characteristics, attributed to the increased contact area between the sensitive layer and gas molecules as well as the compressibility of the sensing layer [29, 30]. 3D MXene spheres with the hollow porous structure meet the above requirements via multiple synergetic strategies. Compared with 2D MXene film, the anti-aggregation structure of porous 3D MXene spheres is very beneficial for gas sensing because of the minimized loss of specific surface area caused by the film aggregation [23, 31]. Porous MXene spheres have substantially more edge defects than MXene sphere, which has shown to significantly increase the gas adsorption capacity, particularly for $\mathrm{NO}_{2}$ [23]. Therefore, porous MXene spheres may have better $\mathrm{NO}_{2}$ selectivity than MXene spheres. Moreover, the hollow structure of the 3D MXene spheres could deform when exposed to external pressure, resulting in changes in electrical conductivity. As a result, all-MXene sensors based on a 3D MXene spheres with a porous hollow structure are expected to achieve highperformance gas and pressure sensing without composite other materials.

Herein, we developed transient all-MXene sensors with gas and pressure sensing capabilities, respectively. The transient gas and pressure sensors employ porous crumpled MXene spheres prepared by ultrasonic spray pyrolysis technology as the sensing layer and polyvinyl alcohol (PVA) substrate embedded with MXene slurry as the electrodes. This fully transient sensor outperforms state-of-the-art studies regarding ultra-wide pressure detection range and ultrahigh sensitivity toward $\mathrm{NO}_{2}$. This work provides another way along the route to wearable and recyclable transient electronics.

\section{Experiment}

\subsection{Preparation of $\mathrm{Ti}_{3} \mathrm{C}_{2} \mathrm{~T}_{x}$ MXene Colloid}

$\mathrm{Ti}_{3} \mathrm{C}_{2} \mathrm{~T}_{\mathrm{x}}$ MXene colloids were synthesized by etching the $\mathrm{Ti}_{3} \mathrm{AlC}_{2}$ phase (Jilin 11 Technology Co., Ltd., China) with 
$\mathrm{LiF} / \mathrm{HCl}$ as reported previously [23]. First, $1 \mathrm{~g} \mathrm{LiF}$ (Aladdin, > 99.99\%) was added into $40 \mathrm{~mL} 12 \mathrm{M} \mathrm{HCl}$ (Xilong Scientific Co., Ltd.) and stirred in a water bath of $40{ }^{\circ} \mathrm{C}$ for $10 \mathrm{~min}$. After $\mathrm{LiF}$ was completely dissolved, $1 \mathrm{~g} \mathrm{Ti}_{3} \mathrm{AlC}_{2}$ was slowly added to the mixed solution, and then, a $40{ }^{\circ} \mathrm{C}$ water bath was conducted for $24 \mathrm{~h}$. After the reaction, the product was centrifugally washed until the supernatant became neutral. Next, the clay $\mathrm{Ti}_{3} \mathrm{C}_{2} \mathrm{~T}_{x}$ was dispersed in an ice bath by ultrasonic treatment for $1 \mathrm{~h}$. Finally, a small amount of $\mathrm{Ti}_{3} \mathrm{C}_{2} \mathrm{~T}_{x}$ dispersion was extracted to make the $\mathrm{Ti}_{3} \mathrm{C}_{2} \mathrm{~T}_{x}$ membrane by vacuum filtration to determine the concentration.

\subsection{Preparation of Polyphenylene (PS) Sphere Colloid}

PS spheres were synthesized by an emulsion-free polymerization method [32]. In brief, $30 \mathrm{~mL}$ styrene (Xilong Scientific Co., Ltd.) and $0.25 \mathrm{~g}$ poly(sodium 4-styrene sulfonic acid) (Aladdin, Mw: 70,000), and $0.15 \mathrm{~g}$ sodium bicarbonate (Aladdin) were dissolved in $300 \mathrm{~mL}$ deionized water and stirred in an oil bath of $70{ }^{\circ} \mathrm{C}$ for $1 \mathrm{~h} .0 .15 \mathrm{~g}$ potassium persulfate (Aladdin, $>99.99 \%$ ) was then added into the solution, and the solution was stirred in a $70{ }^{\circ} \mathrm{C}$ oil bath under $\mathrm{N}_{2}$ atmosphere for $6 \mathrm{~h}$. After the reaction, the white precipitate was thoroughly washed by high-speed centrifugation. Finally, the white precipitate was uniformly dispersed in deionized water using a column ultrasonic machine to obtain a colloid of PS spheres. Similarly, a small amount of PS spheres colloid was extracted to make PS spheres membrane by vacuum filtration to determine the concentration.

\subsection{Preparation of Porous Crumpled MXene Sphere}

Porous crumpled MXene spheres were synthesized by ultrasonic spray pyrolysis technology. Taking 2-5 as an example, the prepared $\mathrm{Ti}_{3} \mathrm{C}_{2} \mathrm{~T}_{\mathrm{x}}$ MXene colloid $\left(10 \mathrm{mg} \mathrm{mL}^{-1}\right)$ and $5 \mathrm{~mL}$ PS sphere colloid (48 $\mathrm{mg} \mathrm{mL}^{-1}$ ) were mixed and diluted by adding deionized water to prepare $50 \mathrm{~mL}$ ultrasonic spray precursor solution, in which the concentration of $\mathrm{Ti}_{3} \mathrm{C}_{2} \mathrm{~T}_{\mathrm{x}}$ MXene was maintained at $2 \mathrm{mg} \mathrm{mL}^{-1}$. The prepared precursor solution was put into the atomization chamber. The fine water mist generated by ultrasound was brought into the tubular furnace preheated to $800{ }^{\circ} \mathrm{C}$ through $\mathrm{Ar}$ gas. Finally, the MS-2-5 powder generated was collected by the electrostatic collector at the back end. For MS-2-10 and MS-2-20, the only difference was that 10 and $20 \mathrm{~mL}$ of PS sphere colloid were added to the precursor solution, respectively.

\subsection{Characterizations}

The morphology of porous crumpled MXene sphere observed by field-emission scanning electron microscopy (FESEM; JEOL JSM-7500F) with an acceleration voltage of $5 \mathrm{kV}$ and transmission electron microscopy (TEM; JEM $2100 \mathrm{~F}$ ) with an acceleration voltage of $200 \mathrm{kV}$. The $\mathrm{X}$-ray diffraction (XRD) patterns of the porous crumpled MXene sphere powder were analyzed using Rigaku D/ Max 2550 with $\mathrm{Cu} \mathrm{K} \alpha$ radiation $(\lambda=1.5418 \AA)$ in the $2 \theta$ range of $3^{\circ}-80^{\circ}$. The surface characteristics of the porous crumpled MXene sphere powder were measured using an ESCALAB $250 \mathrm{X}$-ray photoelectron spectrometer (XPS) with an X-ray source (Al K $\alpha h v=1486.6 \mathrm{eV})$. The specific surface area and pore size distribution of the prepared porous crumpled MXene sphere powder were determined from nitrogen adsorption/desorption isotherms by Brunauer-Emmett-Teller (BET, Micromeritics Gemini VII).

\subsection{Fabrication and Performance Measurement of the Transient $\mathrm{NO}_{2}$ Sensor}

The prepared $\mathrm{Ti}_{3} \mathrm{C}_{2} \mathrm{~T}_{\mathrm{x}}$ MXene colloid was centrifuged at 20,000 rpm for $30 \mathrm{~min}$, and the MXene slurry was precipitated. Masked by the hydrocoagulant film, the MXene slurry was scraped on the glass mold (square groove specification: $21 \times 21 \times 2 \mathrm{~mm}^{3}$ ) to form a cross-finger electrode pattern. The electrode width and electrode spacing were all $1 \mathrm{~mm}$. After the MXene slurry was dried, PVA (0588 low-viscosity) aqueous solution with a concentration of $15 \mathrm{wt} \%$ was slowly dropped into the prepared solution. After natural drying, the PVA film with MXene cross-finger electrode was peeled off. The electrode was masked through the adhesive tape, and then, $5 \mathrm{mg}$ porous crumpled MXene spheres powder was dispersed in $0.1 \mathrm{~mL}$ ethanol and finally dip-coated on the surface of the electrode. After natural drying and removing the mask, the $\mathrm{NO}_{2}$ sensor based on porous crumpled MXene spheres was successfully fabricated. The sensor was placed in the gas cavity of the self-made dynamic test system, where the different concentrations of the target gas were obtained by controlling the ratio of dry air $(80 \%$ 
$\mathrm{N}_{2}$ and $20 \% \mathrm{O}_{2}$ ) and the target gas, with the total flow rate of gas maintained at $500 \mathrm{sccm}$ (standard cubic centimeter per minute). The real-time resistance change of the sensor was detected by connecting the digital multimeter (Fluke $8846 \mathrm{~A}$ ), and the built-in power supply of Fluke 8846A was $5 \mathrm{~V}$ for resistance measurement.

\subsection{Fabrication and Performance Measurement of the Transient Pressure Sensor}

MXene top and bottom electrodes were made in the same way that MXene cross-finger electrodes were made. The square scratching pattern $\left(6 \times 6 \mathrm{~mm}^{2}\right)$ and lead connection were different. After the MXene slurry was dried, the copper wires were connected to the MXene blocks using conductive silver slurry, and then, the PVA solution was poured. $20 \mathrm{mg}$ porous crumpled MXene spheres powder was evenly dispersed in $2 \mathrm{~mL}$ MXene colloid $\left(2 \mathrm{mg} \mathrm{mL}^{-1}\right)$, and then, a round membrane with a diameter of $18 \mathrm{~mm}$ was obtained by vacuum filtration. The membrane was cut into a square $\left(6 \times 6 \mathrm{~mm}^{2}\right)$ as the pressure sensing layer and placed between the top and bottom MXene electrodes. The square sensing layer was tightly encapsulated by brushing PVA aqueous solution on the top and bottom PVA membrane to produce a pressure sensor. Copper wire leads reserved in the top and bottom MXene square electrodes were used for output signals. The pressure-sensing properties were measured by the electromechanical universal testing machine (Mark-10), and the dynamometer model was M5-5. During the pressure test, the sensing area of the pressure sensor was fixed directly below the dynamometer probe about $1 \mathrm{~cm}^{2}$, and the probe completely covers the sensing area of a pressure sensor. The test conditions such as pressure, frequency, and press time can be adjusted by setting maximum force and maximum brake point. The real-time resistance change of the sensor was detected by connecting the digital multimeter (Keithley DMM6500). The pressure response $\left(R_{\mathrm{e}}\right)$ was defined as $\left|\Delta R / R_{0}\right| \times 100 \%$, where $\Delta R$ means the change of the stable resistance between pressure releasing and loading states, and $\mathrm{R}_{0}$ represented the stable resistance of sensor without pressure loading. In addition, $\delta R_{\mathrm{e}} / \delta P$ was recorded as the sensitivity $(S)$ of the pressure sensors, where P represented the intensity of the applied pressure.

\section{Results and Discussion}

The synthetic process of porous crumpled MXene sphere is displayed in Fig. 1a. MXene colloid and PS sphere colloid (grain diameter: $\sim 250 \mathrm{~nm}$, Fig. S1a-b) were evenly mixed in a particular proportion and then atomized into aerosols through an ultrasonic atomizer, which was immediately carried into a high-temperature tubular furnace through highpurity Ar gas. The aerosol encountered high temperatures in the tube furnace, and the moisture vaporized instantly. The disappearance of the internal stress caused the dispersed MXene films and PS spheres in the aerosol to collapse and accumulate inward. At the same time, PS spheres began to decompose when heated and produced $\mathrm{CO}_{2}$ gas, which broke through crumpled MXene spheres and formed a large number of holes on their surface. Porous crumpled MXene sphere powder generated in one step in the tube furnace was then collected by the back-end electrostatic collector. In order to achieve the best porous effect, different proportions of porous crumpled MXene spheres were synthesized by mixing PS spheres with different amounts of MXene, denoted as MS-2-5, MS-2-10, and MS-2-20, respectively.

SEM images of porous crumpled MXene spheres (Fig. 1b-g) indicated the surface morphology became wrinkled, and the size and number of surface holes increased with the increase of PS spheres. Specifically, the round and smooth state in MS-2-5 was transformed into many ridgelike humps on the surface in MS-2-10 and MS-2-20. The resulting ridge-like humps structure was formed by decomposing PS spheres in crumpled MXene spheres, leading to the internal collapse and rupture and holes formed on porous crumpled MXene spheres (Fig. S1f). As the proportion of PS spheres increased, these phenomena became more pronounced. However, when further increase of PS spheres to $20 \mathrm{~mL}$, a large area of the porous crumpled MXene spheres would lose the supporting force, resulting in the collapse of the porous crumpled MXene spheres and the loss of 3D crumpled sphere structure (Fig. S1c-e).

XPS was applied to reveal the chemical states and bonding configurations of porous crumpled MXene spheres. The XPS survey spectra of 2D MXene, MS-2-5, MS-210, and MS-2-20 displayed different Ti, C, O, and F elements signals in porous crumpled MXene spheres (Fig. S2a). The intensity of C $1 \mathrm{~s}$ peak and $\mathrm{O} 1 \mathrm{~s}$ peak rose with the proportion of PS spheres increased, demonstrating the 

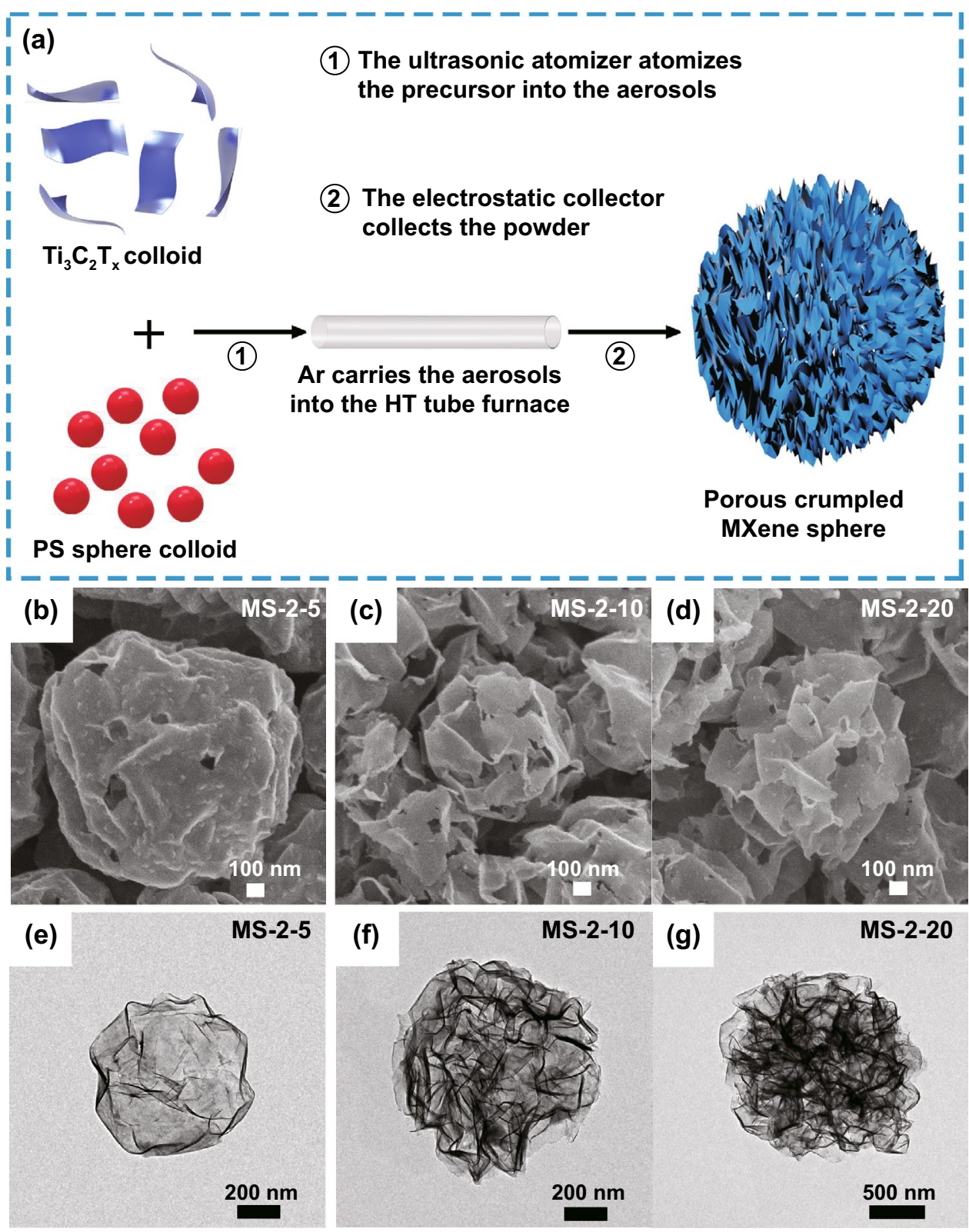

Fig. 1 a The synthesis scheme of porous crumpled MXene spheres. SEM images of b MS-2-5, $\mathbf{c}$ MS-2-10, and d MS-2-20. TEM images of $\mathbf{e}$ MS-2-5, f MS-2-10, and g MS-2-20

decomposition of PS spheres and increase in oxygen terminals. In detail, we measured the high-resolution Ti $2 p$ spectra of 2D MXene, MS-2-5, MS-2-10, and MS-2-20 in Fig. 2a. The Ti $2 \mathrm{p}$ spectra were spin splitting into Ti $2 p_{3 / 2}$ and Ti $2 p_{1 / 2}$ with a distance of $5.8 \mathrm{eV}$. Each splitting peak can be divided into four peaks at $455.57,456.34$, 456.64 , and $458.98 \mathrm{eV}$, corresponding to the four states of $\mathrm{Ti}-\mathrm{C}, \mathrm{Ti}^{2+}, \mathrm{Ti}^{3+}$, and $\mathrm{TiO}_{2}[23]$. The intensity of $\mathrm{TiO}_{2}$ peaks enhanced gradually with more PS spheres due to the increase in oxygen terminals and slight oxidation. The formation of porous crumpled MXene spheres was caused by the destruction of the complete lamellar structure, yielding more edges and defects on MXene. In high-resolution $\mathrm{O}$ $1 \mathrm{~s} \mathrm{spectra}$ of 2D MXene, MS-2-5, MS-2-10, and MS-2-20 (Fig. S2b), each spectrum can be fitted from three peaks at $529.89,531.86$, and $533.23 \mathrm{eV}$, representing $\mathrm{Ti}-\mathrm{O}, \mathrm{O}-\mathrm{H}$, and $\mathrm{C}-\mathrm{O}-\mathrm{C}$, respectively [33-35]. It can be seen that the proportion of $-\mathrm{OH}$ terminal in porous MXene crumpled 
spheres was significantly lower than that in 2D MXene film after high-temperature treatment.

XRD patterns of 2D MXene, MS-2-5, MS-2-10, and MS-2-20 are shown in Fig. 2b. The (002) characteristic diffraction peak of 2D MXene was strong, and the secondary diffraction peak was also prominent [23]. Combined with the XPS spectrum of 2D MXene, it could be indicated that the 2D MXene we prepared with high quality and purity. After forming a porous sphere structure, the intensity of (002) peak decreased significantly, and (110) peak became pronounced due to the increased stacking and random orientation of MXene films. The $\mathrm{N}_{2}$ adsorption/desorption isotherms of porous crumpled MXene sphere displayed typical mesoporous characteristics with the type III isotherm and a type H3 hysteresis loop (Fig. 2c). The isotherm had no inflection point, and the adsorption appeared self-accelerating phenomenon. Moreover, there was no apparent saturated adsorption platform under high pressure, indicating that the pore structure was irregular, consistent with the irregular holes observed in SEM. The pore size observed by SEM was also coincident with the pore size distribution analysis (Fig. 2d). The pore sizes of MS-2-20 and MS-2-10 were concentrated around $100 \mathrm{~nm}$, while the pore sizes of MS-2-5 were mainly distributed around $80 \mathrm{~nm}$. It should be noted that the specific surface area of MXene increases sharply with the generation of the porous sphere structure. The specific surface area of MS-2-5, MS-2-10, and MS-2-20 reached 91.77, 137.80 , and $93.35 \mathrm{~m}^{2} \mathrm{~g}^{-1}$, respectively, compared with 33.56 $\mathrm{m}^{2} \mathrm{~g}^{-1}$ for the dried MXene films as previously reported [23]. The specific surface area of MS-2-20 did not further increase due to the collapse of the sphere structure with excessive PS spheres mentioned above in Fig. S1e.
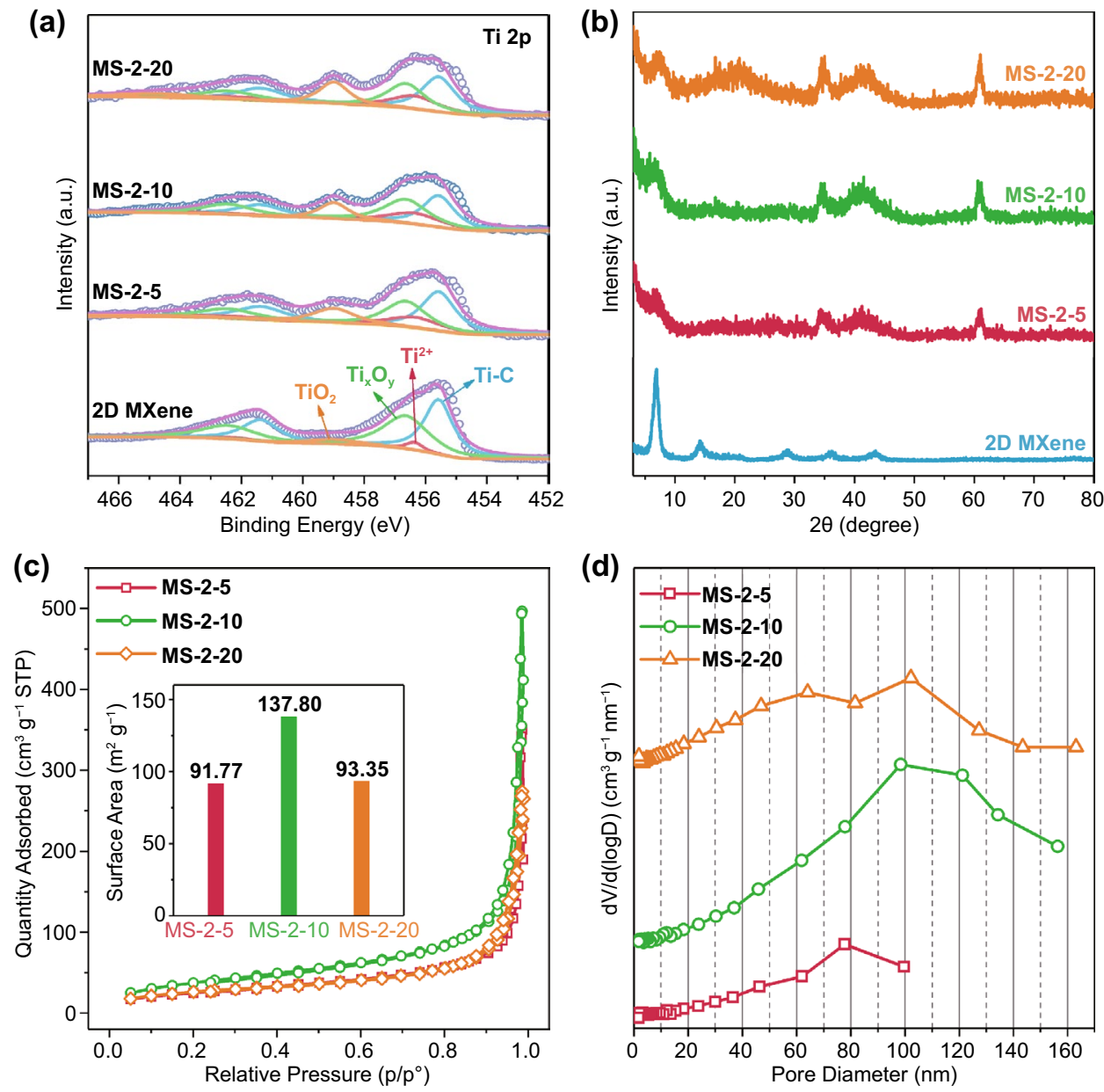

Fig. 2 a Ti 2p XPS spectra and b XRD patterns of 2D MXene, MS-2-5, MS-2-10, and MS-2-20. c N 2 adsorption-desorption isotherms and d pore size distribution of MS-2-5, MS-2-10, and MS-2-20 
The fabrication process of the transient substrate with the MXene interdigital electrode is shown in Fig. 3a. MXene slurry was readily produced by high-speed centrifugation (Fig. 3b), yielding a single layer mixture and few MXene films. The interdigital electrode pattern was fabricated by scraping and coating the MXene slurry on the bottom of the glass mold through the hydrogel mask. Subsequently, PVA aqueous solution (15 wt \%) was coated onto the dried MXene patterned electrode. After dried at room temperature for $24 \mathrm{~h}$, the PVA film with MXene cross-finger electrode (Fig. 3c) was peeled off and clipped to obtain the substrate. We also prepared PVA films with conductive patterns of "MXENE" and "JLU," as shown in Fig. 3d-e, and the detailed production steps are displayed in Fig. S3. As evidenced by the excellent conductivity $(15.1 \Omega)$ of the MXene electrode (Fig. S4a), we confirmed its feasibility as an electrode. The stability of MXene electrodes was tested with polyimide tape (Fig. S4b-d). After removing the adhesive tape, MXene electrodes could stably adhere to the substrate with a resistance change rate of only $1.3 \%$. As shown in Fig. 3f-g, MXene electrodes were embedded in the PVA membrane, enabling robust and highly stable MXene electrodes on the PVA substrate.

The transient $\mathrm{NO}_{2}$ sensors were fabricated by drop-casting porous crumpled MXene spheres powder on the prepared water-soluble PVA substrates embedded with interdigital MXene electrodes (Fig. 3h). The gas-sensing properties were tested by a dynamic test system (Fig. S5). We measured the performance of porous crumpled MXene spheres with different ratios in continuous response to $50 \mathrm{ppb}, 100 \mathrm{ppb}$, $500 \mathrm{ppb}, 1 \mathrm{ppm}$, and 5 ppm $\mathrm{NO}_{2}$ in Fig. 3i. The transient sensor based on MS-2-5 had higher noise and the lowest responses at each concentration among three porous crumpled MXene spheres, while the sensor based on MS-2-20 performed best at a low concentration of $\mathrm{NO}_{2}$. The above results were confirmed by the response values corresponding to different concentrations of $\mathrm{NO}_{2}$ shown in Fig. 3j. When the concentration of $\mathrm{NO}_{2}$ reached $5 \mathrm{ppm}$, the response value of the MS-2-10 sensor remained the highest among the three kinds of porous spheres in multiple tests, reaching $12.11 \%$ at most, which was a considerable improvement compared with previous gas sensors based on MXene film [11, 23]. Meanwhile, the sensor based on MS-2-10 had a low detection limit of $50 \mathrm{ppb}$ with a response of $0.25 \%$.

The repeatability of the sensors was measured by exposing the sensors to 5 ppm $\mathrm{NO}_{2}$ three consecutive times (Fig. 3k). It could be seen that the MS-2-10 sensor maintained the highest response with almost the same response values three times in Fig. 3c. It had an irreversible response to each concentration of $\mathrm{NO}_{2}$ and could maintain the initial response when out of the gas atmosphere to be measured, making sensors very suitable as disposable and discardable $\mathrm{NO}_{2}$ sensors. In Fig. S6, the selectivity of the sensors was investigated by comparing the response of the sensors to $100 \mathrm{ppm}$ ammonia and various volatile organic compounds (VOCs). Although the concentration of the contrast gases was much higher than $5 \mathrm{ppm}$, the response of the sensors based on porous crumpled MXene spheres was much higher to 5 ppm $\mathrm{NO}_{2}$ than to ammonia and various VOCs. Obtained from Fig. 31, the MS-2-10 sensor showed the best $\mathrm{NO}_{2}$ selectivity among the sensors based on porous crumpled MXene spheres, and its responses to ammonia, ethanol, acetone, ethanol, and toluene were $4.35 \%$, $1.99 \%, 1.39 \%, 2.98 \%$, and $0.73 \%$, respectively. It was noteworthy that the MS-2-10 sensor exhibited much higher gas response and lower detection limits than those of the sensors based on MXene films reported in previous work (Fig. 3m) $[11,21,23,36,37]$. The effect of relative humidity on $\mathrm{NO}_{2}$ sensing performance was also investigated at the relative humidity of $0 \%, 30 \%, 60 \%$, and 90\%, respectively (Fig. S7). The response of MS-2-10 to $\mathrm{NO}_{2}$ was improved as humidity increased, indicating that water molecules can promote the adsorption of $\mathrm{NO}_{2}$ molecules to the MXene surface. The excellent gas-sensing performance of MS-2-10 was attributed to its large specific surface area, as well as an abundance of edges and defects generated by folding and porous structure, as previously discussed [23]. MS-2-10 has many adsorption sites for gas molecules because of its large specific surface area, while the rich edge defects greatly enhanced the adsorption capacity of gas molecules, especially for $\mathrm{NO}_{2}$ molecules. The electron transfer between the surface of $\mathrm{MXene}$ and $\mathrm{NO}_{2}$ molecules led to the decrease in carrier concentration and electrical conductivity.

The hollow structure of porous crumpled MXene spheres provided a large specific surface area, which enabled excellent $\mathrm{NO}_{2}$ sensing performance and changed its conductivity under external pressure by readily collapsing the structure. We mixed porous crumpled MXene spheres and MXene films and produced a composite membrane by vacuum filtration, which can obtain the ultra-high pressure-sensing properties that pure MXene membrane does not possess. As shown in Fig. 4a, the transient pressure sensor can be fabricated quickly by encapsulating a porous crumpled 


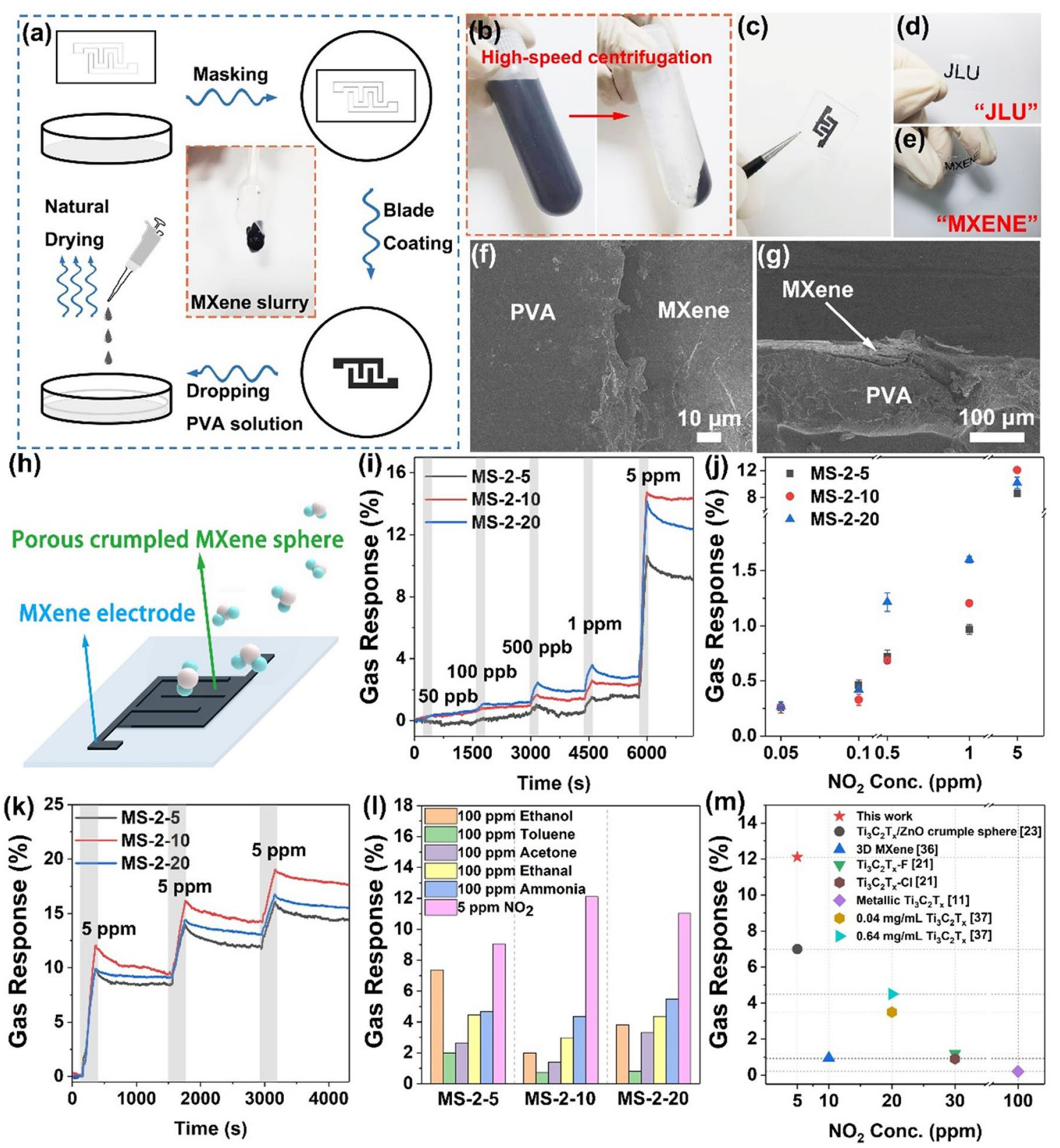

Fig. 3 a Schematic diagram of the fabrication process of the transient PVA substrate with the MXene cross-finger electrode and the photograph of the MXene slurry. b Images of the preparation process of MXene slurry. c Image of the transient PVA substrate with MXene cross-finger electrode. d-e Images of the transient PVA substrate with conductive patterns of "JLU" and "MXENE." $\mathbf{f}$ The frontal SEM image and $\mathbf{g}$ the sectional SEM image of the PVA substrate with MXene cross-finger electrode. $\mathbf{h}$ Schematic diagram of the transient $\mathrm{NO}_{2}$ sensor based on a porous crumpled MXene sphere. i Dynamic response-recovery curve of sensors based on MS-2-5, MS-2-10, and MS-2-20 in the different concentrations of dry $\mathrm{NO}_{2}$. $\mathbf{j}$ Gas response of sensors based on MS-2-5, MS-2-10, and MS-2-20 depending on $\mathrm{NO}_{2}$ concentration. $\mathbf{k}$ Real-time resistance curve of MS-2-5, MS-2-10, and MS-2-20 in response to $5 \mathrm{ppm}$ of $\mathrm{NO}_{2}$ for three consecutive times. $\mathbf{l}$ The maximum resistance change rate of sensors based on MS-2-5, MS-2-10, and MS-2-20 to $100 \mathrm{ppm}$ of ethanol, acetone, ethanol, toluene, $\mathrm{NH}_{3}$, and $5 \mathrm{ppm}$ of $\mathrm{NO}_{2}$. $\mathbf{m}$ Comparison of the performance of MXene-based $\mathrm{NO}_{2}$ sensors

MXene spheres (MS-2-10) composite membrane between the MXene square electrodes. Figure $4 \mathrm{~b}-\mathrm{c}$ displays the cross section morphology of the composite membrane. It shows many porous crumpled MXene spheres among MXene films, producing a larger interspace for pressure regulation than pure MXene membranes (Fig. S8). 
The response values of the sensor under different intensities of pressure are showed in Fig. $4 \mathrm{~d}$. The rate of increase in the response gradually slowed down as the intensity of pressure increased. The sensors maintained the highest linear sensitivity of $3.14 \mathrm{kPa}^{-1}$ in the range of 0.14 to $22.22 \mathrm{kPa}$ and only $0.22 \mathrm{kPa}^{-1}$ in the range of $22.22-140 \mathrm{kPa}$. In a small pressure range, the external force applied to the sensor deformed porous MXene crumpled spheres, increasing the conductive path above and below porous MXene crumpled spheres and lowing the resistance of pressure sensors. When the pressure reached a large pressure range, however, porous MXene crumpled spheres cannot be further compressed as the pressure rises. The applied pressure can only further reduce the interspace between MXene films and porous MXene crumpled spheres, but the compression was more difficult and the variation was tiny. So the sensitivity of the pressure sensor was divided into two parts as the pressure increased. Figure $4 \mathrm{e}$ shows the real-time change of the resistance at different pressure intensities. When the same intensity of pressure was loaded three times in a row, the response was almost the same and enhanced with increasing the intensity of pressure. Specifically, a saturated response value of 91.56 was achieved at $555.56 \mathrm{kPa}$, while a response of 2.22 was obtained at a very low pressure of $140 \mathrm{~Pa}$, indicating an outstanding ultra-wide range of pressure tests. Thus, the sensitivity of pressure sensors based on porous crumpled MXene spheres was not outstanding because the pressure load would reduce the sensor's resistance, and the response range can only be measured between 0 and 100 . However, pressure sensor based on porous crumpled MXene spheres shows the widest linear detection range of $0.14-22.22 \mathrm{kPa}$ (Fig. 4f) compared to the previously reported pressure sensors based on MXene and graphene [14, 38-45]. We tested the effect of load residence times on the sensor of $0.1,0.5$, 1.0, 2.0, and $5.0 \mathrm{~s}$. As shown in Fig. S9, the width of the response peak of the real-time resistance curve increased as the loading time lengthened.

Meanwhile, we measured the effect of loading frequency on the performance of sensors under $22.22 \mathrm{kPa}$ pressure load (Fig. S10). With the increase in loading frequency, the response value remained stable to be about 52.5, which proved that the sensor could work stably under different pressure loading frequencies. Immediately afterward, we carried out an anti-fatigue test on the sensor. By continuously applying a load of $41.67 \mathrm{kPa} 1000$ times, we observed the response was consistently stable around 59 (Fig. 4g), confirming a stable and robust operation even in multiple consecutive tests. The accurate response to the loading times again demonstrated the high sensitivity of the sensors to pressure loads. The response time (defined as the time required during 10-90\% of the stable resistance change between pressure releasing and loading states) was only $34 \mathrm{~ms}$ at a load of $55.56 \mathrm{kPa}$ (Fig. 4h), ensuring a real-time sensing response to the pressure load.

The pressure sensor in the actual application scenarios was examined when the experimenter presses manually in the low-pressure range in Fig. 5a. The sensor was attached to the wrist of the 25-year-old experimenter through the medical polyurethane (PU) membrane (inset of Fig. 5b) to record radial artery blood pressure. Figure $5 b$ displayed the regular and repetitive waveforms of the wrist pulse with a periodic beating of 96 beats per minute, where the characteristic systolic peak $(P 1)$ and diastolic peak $(P 2)$ were observed [46, 47]. Another proven application was the detection of tiny vibrations by sensors. In Fig. 5c, we used the pressure sensors to detect the different vibration patterns of the mobile phone ("Off-beat," "Ripple," "Waltz" and "Zig-Zig-Zig" vibration patterns in Samsung S10 plus). The phone was placed flat on the sensor with the sensor in the center of the phone. Then, the experimenter pressed different vibration modes, which were automatically repeated three times (Video S1). It can be observed that the signal waveform of the sensor output was highly consistent with the vibration sound waveform in Fig. 5d-g, which confirmed the capability to respond to tiny vibrations. Based on the performance of sensors, we aggressively tried to simulate the sensors as an electronic throat to receive the sound signal by detecting the motion of the throat and the vibration of the vocal cords [48]. Similarly, the sensor was tightly attached to the throat of the experimenter (Fig. 5h), and then, the experimenter spoke three words ("degradable," "MXene" and "sensor") as smoothly as possible and repeated each word three times. In Fig. 5i-k, as a triphthong word, the response signal waveform of the word "degradable" had three peaks, while as diphthong words, the response signal waveform of words "MXene" and "sensor" had only two peaks. In addition, because the pronunciation of the word "MXene" brought greater throat peristalsis than the word "sensor," the response signal waveform of the word "MXene" was more undulating than that of the word "sensor." Therefore, the sensors can predict the speech content by recording throat peristalsis and vocal cord vibration to a certain extent. After 
(a)

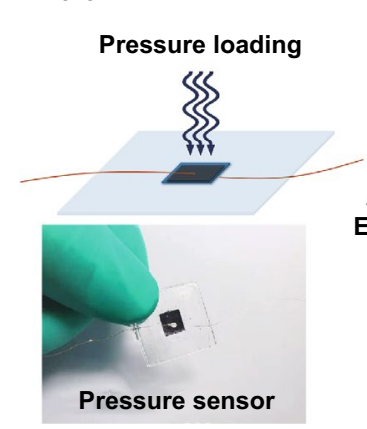

(d) 100
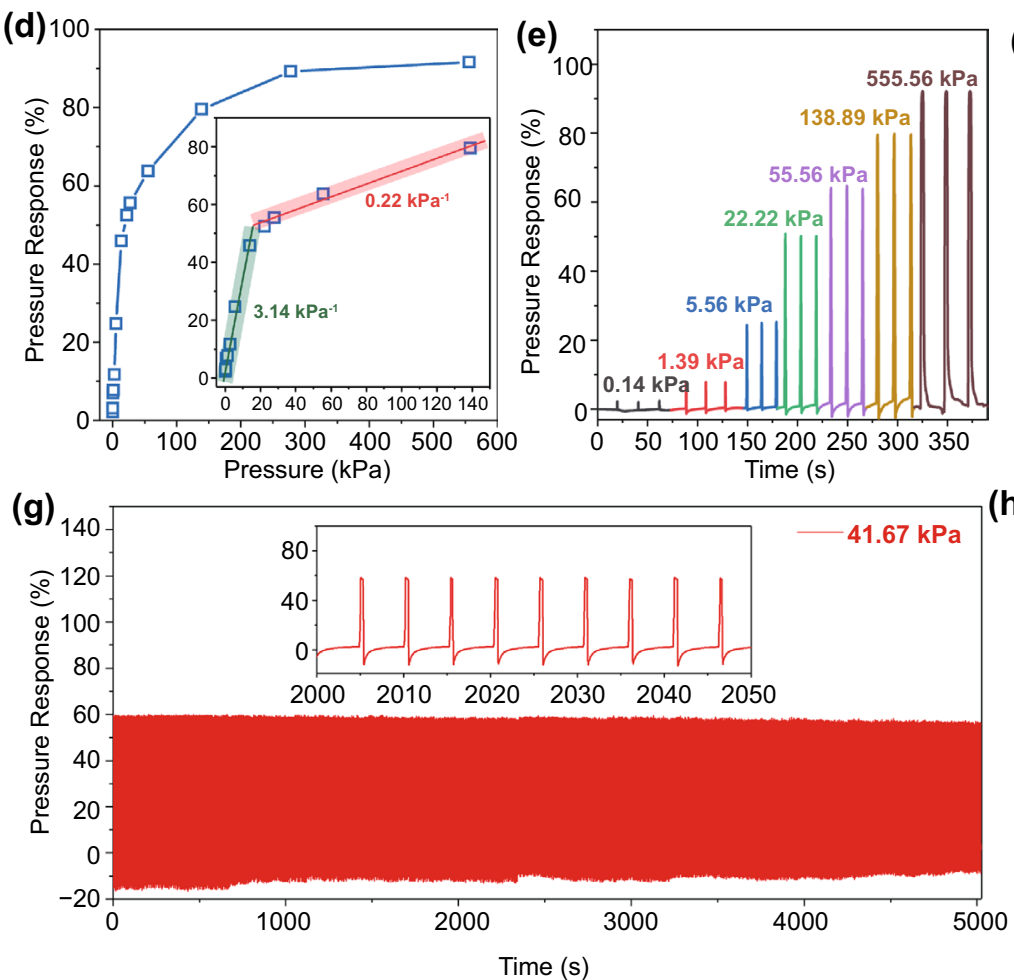
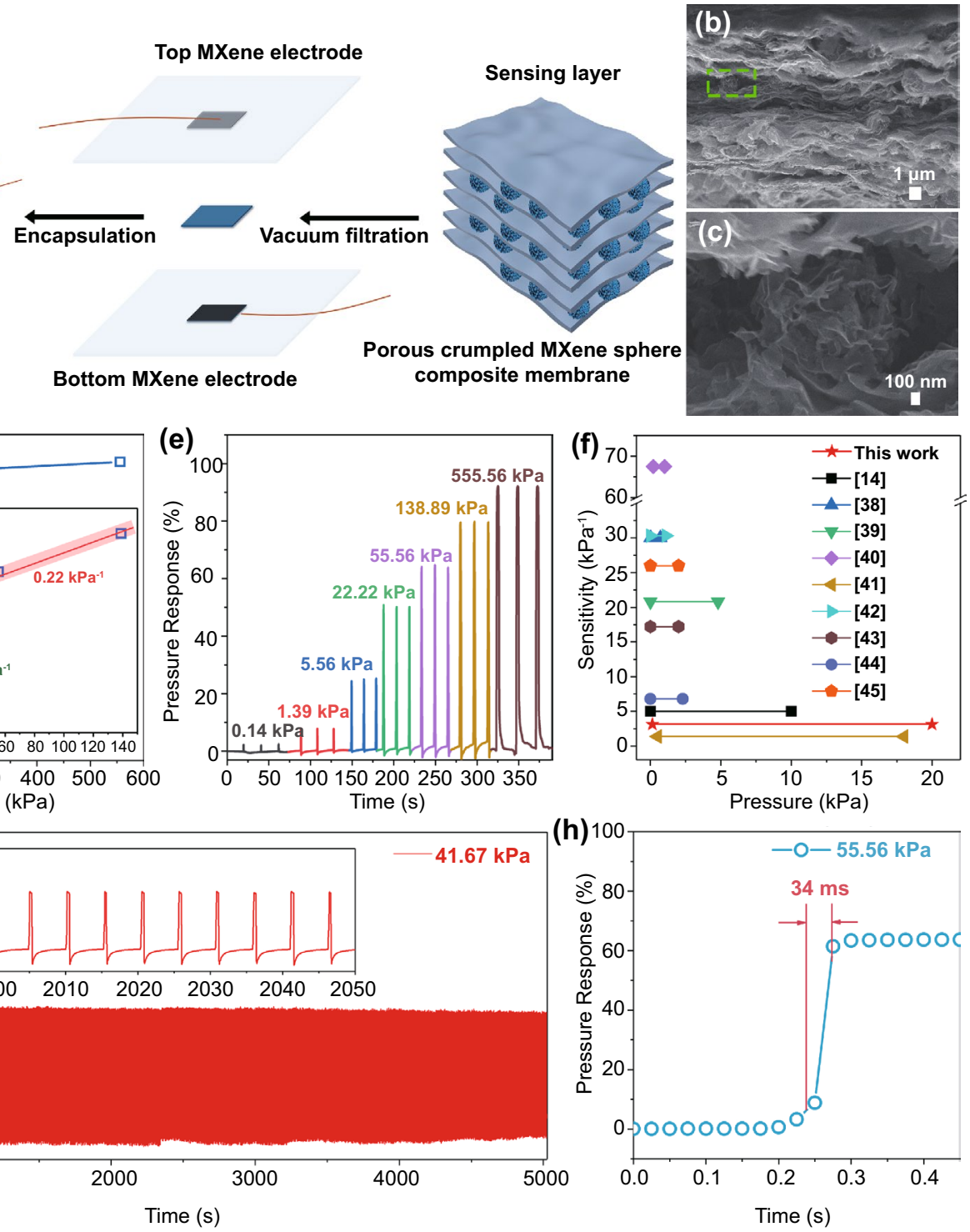

Fig. 4 a Schematic diagram of the pressure sensor fabrication based on MS-2-10 and a photograph of the fabricated pressure sensor. b The cross section SEM image of the composite membrane and $\mathbf{c}$ the close-up image of porous crumpled MXene sphere in the green box. $\mathbf{d}$ Pressure response value curve and sensitivity with pressure, inset shows the linear range of pressure response curve. e Real-time resistance curve at different pressures. $\mathbf{f}$ The performance comparison of resistive pressure sensors based on MXene and graphene. g Real-time resistance curve of $41.67 \mathrm{kPa}$ pressure load with 1000 recycles, inset shows the enlarged curve from 2000 to 2050s. h Pressure response time at a loading pressure of $55.56 \mathrm{kPa}$

further improvement and system integration, it is expected to help some mute people.

In order to study the degradable performance of the transient all-MXene gas and pressure sensors based on porous crumpled MXene spheres, the sensors were placed in watch glasses with $50 \mathrm{~mL} \mathrm{H}_{2} \mathrm{O}_{2}$ of different concentrations, and the state of the sensors was continuously observed and recorded. Figure 6a displays the degradation process of the gas and pressure sensor in $2 \%$ medical-grade $\mathrm{H}_{2} \mathrm{O}_{2}$. The PVA substrate was rapidly dissolved within $60 \mathrm{~min}$, while porous crumpled MXene spheres and MXene electrodes were also slowly disappeared after $6 \mathrm{~h}$ degradation with the help of $\mathrm{H}_{2} \mathrm{O}_{2}$ in Fig. 6b. The pressure sensors showed a faster fulldegradation $(4 \mathrm{~h})$ than the gas sensor in $2 \%$ medical-grade of $\mathrm{H}_{2} \mathrm{O}_{2}$ (Fig. 6c). The sensors could be rapidly degraded in $10 \%$ and $30 \% \mathrm{H}_{2} \mathrm{O}_{2}$ for only 30 and $60 \mathrm{~min}$, respectively 

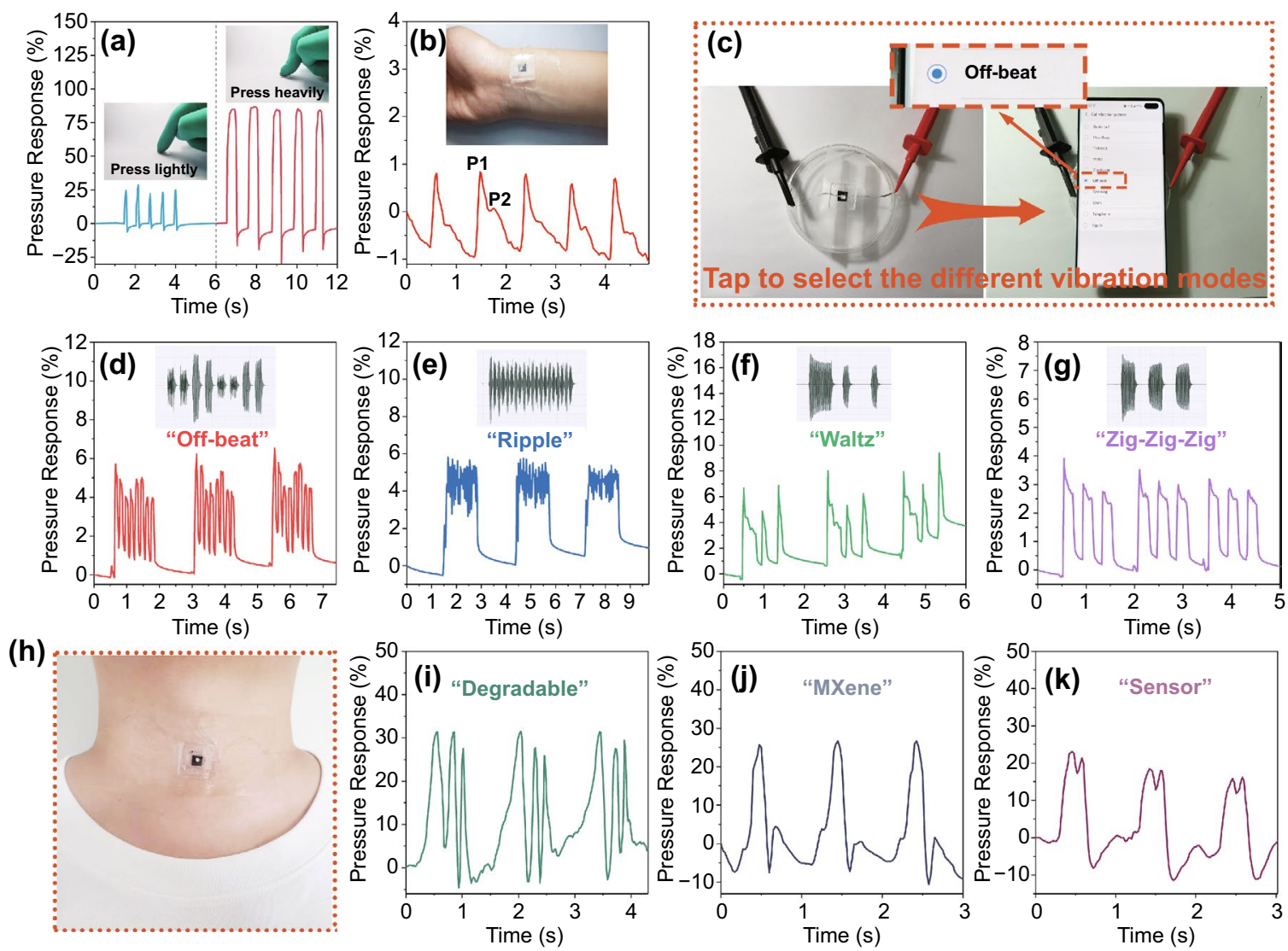

Fig. 5 a The response results of manual press on the sensor. b Real-time recording of the radial artery blood pressure waveform, inset shows the photograph of the wearable sensor attached to the human wrist. $\mathbf{c}$ Photos of the vibration test of the mobile phone (Samsung S10 Plus). Realtime resistance changes of the pressure sensor during detecting the four different vibration modes of the mobile phone: $\mathbf{d}$ "Off-beat," $\mathbf{e}$ "Ripple" and $\mathbf{f}$ "Waltz" and $\mathbf{g}$ "Zig-Zig-Zig." $\mathbf{h}$ The photo of the pressure sensor in the throat. The response signal waveform of the pressure sensor toward sounds: i "degradable," $\mathbf{j}$ "MXene," and $\mathbf{k}$ "Sensor"

(Fig. S11), indicating their capability toward controllable degradation. These results fully proved that the transient allMXene gas and pressure sensors were degradable without any environmental footprint.

\section{Conclusions}

In summary, we have demonstrated the transient allMXene sensors based on porous crumpled MXene spheres, which took advantage of the unique properties of MXene and the novel structure to achieve excellent gas- and pressure-sensitive performance, respectively. The fabricated $\mathrm{NO}_{2}$ sensor exhibited high selectivity, low detection limit (50 ppb), and high response ( $12.11 \%$ to $5 \mathrm{ppm} \mathrm{NO}_{2}$ ). The assembled pressure sensor showed an ultra-wide linear detection range of $0.14-22.22 \mathrm{kPa}$ with a sensitivity of $3.14 \mathrm{kPa}^{-1}$, fast response (34 ms), and excellent repeatability (over 1000 cycles). The pressure sensor attached to the human body can effectively detect the physiological data signals from the wrist pulse signal and the throat vibration during speaking. The multi-functional wearable sensors show excellent controllable transiency with a degradation profile within $6 \mathrm{~h}$ in medical $\mathrm{H}_{2} \mathrm{O}_{2}(2 \%)$. These results highlight the feasibility of realizing wearable multi-functional all-MXene sensors, thus promoting the application of MXene under multiple scenarios and providing new ways to manufacture high-performance, wearable, and degradable sensors required for the Body Internet of Things. 
(a)
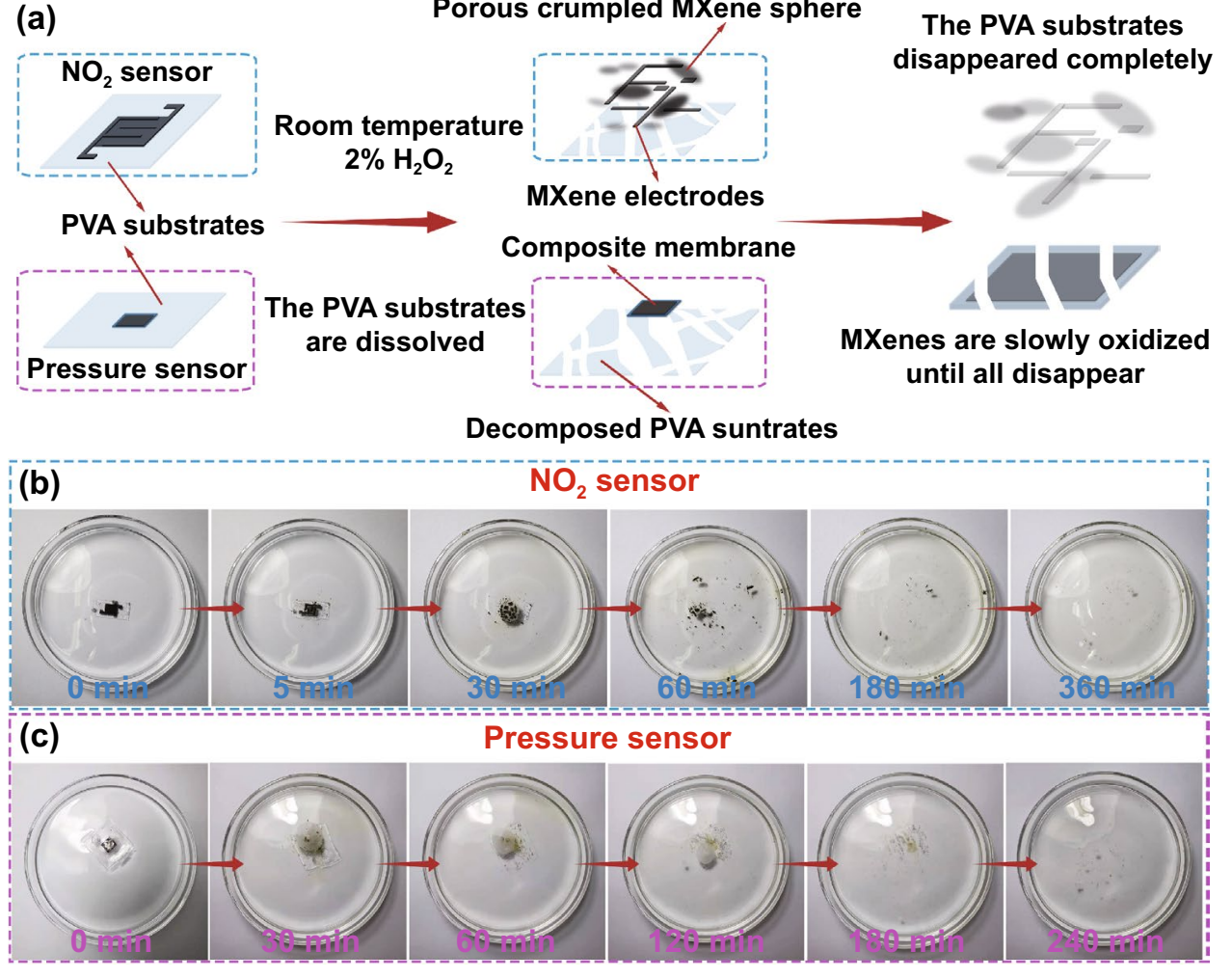

Fig. 6 a Schematic diagram of the degradation process of the transient $\mathrm{NO}_{2}$ and pressure sensors based on MS-2-10. b The degradation process of the transient $\mathrm{NO}_{2}$ sensor in $2 \%$ medical $\mathrm{H}_{2} \mathrm{O}_{2}$ for $6 \mathrm{~h}$. c The degradation process of the transient pressure sensor in $2 \%$ medical $\mathrm{H}_{2} \mathrm{O}_{2}$ for $4 \mathrm{~h}$

Acknowledgements This work is supported by the National Nature Science Foundation of China (Nos. 62122030, 61831011, 61803171, 61722305, 61833006, and 21902057), Program for Chang Jiang Scholars and Innovative Research Team in University (No. IRT-17R47), Application and Basic Research of Jilin Province (20130102010 JC), Young Elite Scientists Sponsorship Program by CAST (2018QN RC001), Jilin Province Science and Technology Development Plan Program (20200301010RQ), Fundamental Research Funds for the Central Universities, Graduate Innovation Fund of Jilin University (101832020CX170).

Funding Open access funding provided by Shanghai Jiao Tong University.

Open Access This article is licensed under a Creative Commons Attribution 4.0 International License, which permits use, sharing, adaptation, distribution and reproduction in any medium or format, as long as you give appropriate credit to the original author(s) and the source, provide a link to the Creative Commons licence, and indicate if changes were made. The images or other third party material in this article are included in the article's Creative Commons licence, unless indicated otherwise in a credit line to the material. If material is not included in the article's Creative Commons licence and your intended use is not permitted by statutory regulation or exceeds the permitted use, you will need to obtain permission directly from the copyright holder. To view a copy of this licence, visit http://creativecommons.org/licenses/by/4.0/.

Supplementary Information The online version contains supplementary material available at https://doi.org/10.1007/ s40820-022-00796-7.

\section{References}

1. D. Helbing, Globally networked risks and how to respond. Nature 497, 51-59 (2013). https://doi.org/10.1038/natur e12047

2. Y. Zhang, Q. Niu, X. Gu, N. Yang, G. Zhao, Recent progress on carbon nanomaterials for the electrochemical detection and removal of environmental pollutants. Nanoscale 11(25), 11992-12014 (2019). https://doi.org/10.1039/C9NR02935D

3. K. Song, P. Xu, G. Wei, Y. Chen, Q. Wang, Health management decision of sensor system based on health reliability degree and grey group decision-making. Sensors 18(7), 2316 (2018). https://doi.org/10.3390/s18072316 
4. J. Lepawsky, Sources and streams of electronic waste. One Earth 3(1), 13-16 (2020). https://doi.org/10.1016/j.oneear. 2020.07.001

5. K. Zhang, J. Schnoor, E. Zeng, E-waste recycling: where does it go from here? Environ. Sci. Technol. 46(20), 10861-10867 (2012). https://doi.org/10.1021/es303166s

6. X. Yu, W. Shou, B.K. Mahajan, X. Huang, H. Pan, Materials, processes, and facile manufacturing for bioresorbable electronics: a review. Adv. Mater. 30(28), e1707624 (2018). https://doi.org/10.1002/adma.201707624

7. X. Zhuang, D. Zhang, X. Wang, X. Yu, J. Yu, Biocompatible and degradable gelatin dielectric based low-operating voltage organic transistors for ultra-high sensitivity $\mathrm{NH}_{3}$ detection. Appl. Phys. Lett. 113(26), 263301 (2018). https://doi.org/10. 1063/1.5054026

8. Y. Guo, S. Chen, L. Sun, L. Yang, L. Zhang et al., Degradable and fully recyclable dynamic thermoset elastomer for 3D-printed wearable electronics. Adv. Funct. Mater. 31(9), 2009799 (2020). https://doi.org/10.1002/adfm.202009799

9. S. Zheng, R. Du, N. Wang, M. Cao, Y. Zhang et al., Construction of dual conductive network in paper-based composites towards flexible degradable dual-mode sensor. Compos. Part A Appl. Sci. Manuf. 151, 106649 (2021). https://doi.org/10. 1016/j.compositesa.2021.106649

10. X. Jin, L. Li, S. Zhao, X. Li, K. Jiang et al., Assessment of occlusal force and local gas release using degradable bacterial cellulose/ $\mathrm{Ti}_{3} \mathrm{C}_{2} \mathrm{~T}_{\mathrm{x}}$ MXene bioaerogel for oral healthcare. ACS Nano 15(11), 18385-18393 (2021). https://doi.org/10.1021/ acsnano.1c07891

11. S.J. Kim, H.J. Koh, C.E. Ren, O. Kwon, K. Maleski et al., Metallic $\mathrm{Ti}_{3} \mathrm{C}_{2} \mathrm{~T}_{\mathrm{x}}$ MXene gas sensors with ultrahigh signalto-noise ratio. ACS Nano 12(2), 986-993 (2018). https://doi. org/10.1021/acsnano.7b07460

12. L. Li, X. Fu, S. Chen, S. Uzun, A.S. Levitt et al., Hydrophobic and stable MXene-polymer pressure sensors for wearable electronics. ACS Appl. Mater. Interfaces 12(13), 15362-915369 (2020). https://doi.org/10.1021/acsami.0c00255

13. Y. Ma, Y. Yue, H. Zhang, F. Cheng, W. Zhao et al., 3D synergistical MXene/reduced graphene oxide aerogel for a piezoresistive sensor. ACS Nano 12(4), 3209-93216 (2018). https://doi.org/10.1021/acsnano.7b06909

14. H.J. Lee, J.C. Yang, J. Choi, J. Kim, G.S. Lee et al., Heterodimensional 2D $\mathrm{Ti}_{3} \mathrm{C}_{2} \mathrm{~T}_{\mathrm{x}}$ MXene and $1 \mathrm{D}$ graphene nanoribbon hybrids for machine learning-assisted pressure sensors. ACS Nano 15(6), 10347-910356 (2021). https://doi.org/10. 1021/acsnano.1c02567

15. C. Zhang, L. McKeon, M. Kremer, S. Park, O. Ronan et al., Additive-free MXene inks and direct printing of microsupercapacitors. Nat. Commun. 10, 1795 (2019). https:// doi.org/10.1038/s41467-019-09398-1

16. S. Abdolhosseinzadeh, R. Schneider, A. Verma, J. Heier, F. Nuesch et al., Turning trash into treasure: additive free MXene sediment inks for screen-printed micro-supercapacitors. Adv. Mater. 32(17), 2000716 (2020). https://doi.org/ 10.1002/adma.202000716
17. X. Wu, T. Tu, Y. Dai, P. Tang, Y. Zhang et al., Direct ink writing of highly conductive MXene frames for tunable electromagnetic interference shielding and electromagnetic wave-induced thermochromism. Nano-Micro Lett. 13, 148 (2021). https://doi.org/10.1007/s40820-021-00665-9

18. R. Kumar, X. Liu, J. Zhang, M. Kumar, Room-temperature gas sensors under photoactivation: from metal oxides to $2 \mathrm{D}$ materials. Nano-Micro Lett. 12, 164 (2020). https://doi.org/ 10.1007/s40820-020-00503-4

19. Y. Guo, M. Zhong, Z. Fang, P. Wan, G. Yu, A wearable transient pressure sensor made with MXene nanosheets for sensitive broad-range human-machine interfacing. Nano Lett. 19(2), 1143-1150 (2019). https://doi.org/10.1021/acs. nanolett.8b04514

20. Q. Yang, Z. Huang, X. Li, Z. Liu, H. Li et al., A wholly degradable, rechargeable $\mathrm{Zn}-\mathrm{Ti}_{3} \mathrm{C}_{2} \mathrm{MXene}$ capacitor with superior anti-self-discharge function. ACS Nano 13(7), 8275-8283 (2019). https://doi.org/10.1021/acsnano.9b03650

21. W. Chen, S. Lai, C. Yen, X. Jiang, D. Peroulis et al., Surface functionalization of $\mathrm{Ti}_{3} \mathrm{C}_{2} \mathrm{~T}_{\mathrm{x}}$ MXene with highly reliable superhydrophobic protection for volatile organic compounds sensing. ACS Nano 14(9), 11490-11501 (2020). https://doi. org/10.1021/acsnano.0c03896

22. Z. Yang, A. Liu, C. Wang, F. Liu, J. He et al., Improvement of gas and humidity sensing properties of organ-like MXene by alkaline treatment. ACS Sens. 4(5), 1261-1269 (2019). https:// doi.org/10.1021/acssensors.9b00127

23. Z. Yang, L. Jiang, J. Wang, F. Liu, J. He et al., Flexible resistive $\mathrm{NO}_{2}$ gas sensor of three-dimensional crumpled MXene $\mathrm{Ti}_{3} \mathrm{C}_{2} \mathrm{~T}_{\mathrm{x}} / \mathrm{ZnO}$ spheres for room temperature application. Sens. Actuators B Chem. 326, 128828 (2021). https://doi.org/10. 1016/j.snb.2020.128828

24. W.Y. Chen, X. Jiang, S.N. Lai, D. Peroulis, L. Stanciu, Nanohybrids of a MXene and transition metal dichalcogenide for selective detection of volatile organic compounds. Nat. Commun. 11, 1302 (2020). https://doi.org/10.1038/ s41467-020-15092-4

25. D. Wang, L. Wang, Z. Lou, Y. Zheng, K. Wang et al., Biomimetic, biocompatible and robust silk fibroin-MXene film with stable 3D cross-link structure for flexible pressure sensors. Nano Energy 78, 105252 (2020). https://doi.org/10.1016/j. nanoen.2020.105252

26. S. Sharma, A. Chhetry, S. Zhang, H. Yoon, C. Park et al., Hydrogen-bond-triggered hybrid nanofibrous membranebased wearable pressure sensor with ultrahigh sensitivity over a broad pressure range. ACS Nano 15(3), 4380-4393 (2021). https://doi.org/10.1021/acsnano.0c07847

27. Y. Yue, N. Liu, W. Liu, M. Li, Y. Ma et al., 3D hybrid porous MXene-sponge network and its application in piezoresistive sensor. Nano Energy 50, 79-87 (2018). https://doi.org/10. 1016/j.nanoen.2018.05.020

28. K. Wang, Z. Lou, L. Wang, L. Zhao, S. Zhao et al., Bioinspired interlocked structure-induced high deformability for two-dimensional titanium carbide (MXene)/natural 
microcapsule-based flexible pressure sensors. ACS Nano 13(8), 9139-9147 (2019). https://doi.org/10.1021/acsnano. $9 \mathrm{~b} 03454$

29. Y. Lv, W. Zhan, Y. He, Y. Wang, X. Kong et al., MOF-templated synthesis of porous $\mathrm{Co}_{3} \mathrm{O}_{4}$ concave nanocubes with high specific surface area and their gas-sensing properties. ACS Appl. Mater. Interfaces 6(6), 4186-4195 (2014). https:// doi.org/10.1021/am405858v

30. Y. Zhai, Y. Yu, K. Zhou, Z. Yun, W. Huang et al., Flexible and wearable carbon black/thermoplastic polyurethane foam with a pinnate-veined aligned porous structure for multi-functional piezoresistive sensors. Chem. Eng. J. 382, 1222985 (2020). https://doi.org/10.1016/j.cej.2019.122985

31. L. Xiu, Z. Wang, M. Yu, X. Wu, J. Qiu, Aggregation-resistant 3D MXene-based architecture as efficient bifunctional electrocatalyst for overall water splitting. ACS Nano 12(8), 8017-8028 (2018). https://doi.org/10.1021/acsnano.8b02849

32. J. Yoon, S.H. Choi, J. Kim, H.W. Jang, Y.C. Kang et al., Trimodally porous $\mathrm{SnO}_{2}$ nanospheres with three-dimensional interconnectivity and size tunability: a one-pot synthetic route and potential application as an extremely sensitive ethanol detector. NPG Asia Mater. 8, e244 (2016). https://doi.org/10. 1038/am.2016.16

33. N. Joshi, L.F. Silva, H.S. Jadhavd, F.M. Shimizua, P.H. Sumane et al., Yolk-shelled $\mathrm{ZnCo}_{2} \mathrm{O}_{4}$ microspheres: surface properties and gas sensing application. Sens. Actuators B Chem. 257, 906-915 (2018). https://doi.org/10.1016/j.snb. 2017.11.041

34. R.K. Mishra, G.J. Choi, Y. Sohn, S.H. Lee, J.S. Gwag, A novel RGO/N-RGO supercapacitor architecture for a wide voltage window, high energy density and long-life via voltage holding tests. Chem. Commun. 56(19), 2893-2896 (2020). https://doi. org/10.1039/D0CC00249F

35. R. Wang, Y. Lin, P. Chen, H. Chen, W. Chiu, Anomalous output performance enhancement of RGO-based triboelectric nanogenerators by $\mathrm{Cu}$-bonding. Nano Energy 86, 106126 (2021). https://doi.org/10.1016/j.nanoen.2021.106126

36. W. Yuan, K. Yang, H. Peng, F. Li, F. Yin, A flexible VOCs sensor based on a 3D Mxene framework with a high sensing performance. J. Mater. Chem. A 6(37), 18116-18124 (2018). https://doi.org/10.1039/C8TA06928J

37. Y. Jian, D. Qu, L. Guo, Y. Zhu, C. Su et al., The prior rules of designing $\mathrm{Ti}_{3} \mathrm{C}_{2} \mathrm{~T}_{\mathrm{x}}$ MXene-based gas sensors. Front. Chem. Sci. Eng. 15, 505-517 (2021). https://doi.org/10.1007/ s11705-020-2013-y

38. K.H. Kim, S.K. Hong, N.S. Jang, S.H. Ha, H.W. Lee et al., Wearable resistive pressure sensor based on highly flexible carbon composite conductors with irregular surface morphology. ACS Appl. Mater. Interfaces 9(20), 17499_ 17507 (2017). https://doi.org/10.1021/acsami.7b06119

39. Q. Li, R. Yin, D. Zhang, H. Liu, X. Chen et al., Flexible conductive MXene/cellulose nanocrystal coated nonwoven fabrics for tunable wearable strain/pressure sensors. J. Mater. Chem. A 8(40), 21131-21141 (2020). https://doi.org/10.1039/D0TA0 $7832 \mathrm{H}$

40. L. Wang, M. Zhang, B. Yang, J. Tan, X. Ding, Highly compressible, thermally stable, light-weight, and robust aramid nanofibers $/ \mathrm{Ti}_{3} \mathrm{AlC}_{2} \mathrm{MXene}$ composite aerogel for sensitive pressure sensor. ACS Nano 14(8), 10633-10647 (2020). https://doi.org/10.1021/acsnano.0c04888

41. F. He, X. You, H. Gong, Y. Yang, T. Bai et al., Stretchable, biocompatible, and multi-functional silk fibroin-based hydrogels toward wearable strain/pressure sensors and triboelectric nanogenerators. ACS Appl. Mater. Interfaces 12(5), 6442 6450 (2020). https://doi.org/10.1021/acsami.9b19721

42. Y. Pang, K. Zhang, Z. Yang, S. Jiang, Z. Ju et al., Epidermis microstructure inspired graphene pressure sensor with random distributed spinosum for high sensitivity and large linearity. ACS Nano 12(3), 2346-2354 (2018). https://doi.org/10.1021/ acsnano.7b07613

43. L.Q. Tao, K.N. Zhang, H. Tian, Y. Liu, D.Y. Wang et al., Graphene-paper pressure sensor for detecting human motions. ACS Nano 11(9), 8790-8795 (2017). https://doi.org/10.1021/ acsnano.7b02826

44. X. Wu, Y. Han, X. Zhang, Z. Zhou, C. Lu, Large-area compliant, low-cost, and versatile pressure-sensing platform based on microcrack-designed carbon black@ polyurethane sponge for human-machine interfacing. Adv. Funct. Mater. 26(34), 6246-6256 (2016). https://doi.org/10.1002/adfm.201601995

45. H.B. Yao, J. Ge, C.F. Wang, X. Wang, W. Hu et al., A flexible and highly pressure-sensitive graphene-polyurethane sponge based on fractured microstructure design. Adv. Mater. 25(46), 6692-6698 (2013). https://doi.org/10.1002/adma.201303041

46. W.W. Nichols, Clinical measurement of arterial stiffness obtained from noninvasive pressure waveforms. Am. J. Hypertens. 18, 3S-10S (2005). https://doi.org/10.1016/j.amjhyper. 2004.10.009

47. C. Pang, J.H. Koo, A. Nguyen, J.M. Caves, M.G. Kim et al., Highly skin-conformal microhairy sensor for pulse signal amplification. Adv. Mater. 27(4), 634-640 (2015). https://doi. org/10.1002/adma.201403807

48. Y. Wei, Y. Qiao, G. Jiang, Y. Wang, F. Wang et al., A wearable skinlike ultra-sensitive artificial graphene throat. ACS Nano 13(8), 8639-8647 (2019). https://doi.org/10.1021/acsnano. $9 b 03218$ 